\title{
Serum Prolactin Level to Tumor Size Ratio as a Potential Parameter for Preoperative Differentiation of Prolactinomas from Hyperprolactinemia-Causing Non-Functional Pituitary Adenomas.
}

\section{Jeong-Hwa Kim}

Samsung Medical Center, Sungkyunkwan University School of Medicine

Kyu-Yeon Hur

Samsung Medical Center, Sungkyunkwan University School of Medicine

\section{Sang-Duk Hong}

Samsung Medical Center, Sungkyunkwan University School of Medicine Jung-Won Choi

Samsung Medical Center, Sungkyunkwan University School of Medicine Ho-Jun Seol

Samsung Medical Center, Sungkyunkwan University School of Medicine Do-Hyun Nam

Samsung Medical Center, Sungkyunkwan University School of Medicine Jung-II Lee

Samsung Medical Center, Sungkyunkwan University School of Medicine Doo-Sik Kong ( $\nabla$ neurokong@gmail.com )

Samsung Medical Center, Sungkyunkwan University School of Medicine

\section{Research Article}

Keywords: Hyperprolactinemia, prolactinoma, non-functioning pituitary adenomas, transsphenoidal surgery, pituitary surgery

Posted Date: August 13th, 2021

DOl: https://doi.org/10.21203/rs.3.rs-777986/v1

License: (9) (1) This work is licensed under a Creative Commons Attribution 4.0 International License. Read Full License 
Version of Record: A version of this preprint was published at World Neurosurgery on December 1st, 2021. See the published version at https://doi.org/10.1016/j.wneu.2021.12.074. 


\section{Serum prolactin level to tumor size ratio as a potential parameter for preoperative differentiation of prolactinomas from hyperprolactinemia- causing non-functional pituitary adenomas.}

Jeong-Hwa Kim, $\mathrm{MD}^{1}$ (https://orcid.org/0000-0002-8587-8546), Kyu-Yeon Hur, MD, PhD ${ }^{2}$, Sang-Duk Hong, MD, $\mathrm{PhD}^{3}$, Jung-Won Choi, MD, $\mathrm{PhD}^{1}$, Ho-Jun Seol, MD, $\mathrm{PhD}^{1}$ (https://orcid.org/0000-0003-4187054X), Do-Hyun Nam, MD, PhD ${ }^{1}$, Jung-Il Lee, MD, PhD¹ (https://orcid.org/0000-0001-8143-5513), DooSik Kong ${ }^{*}, \mathrm{MD}, \mathrm{PhD}^{1}$ (https://orcid.org/0000-0002-7519-3594)

${ }^{1}$ Department of Neurosurgery, Samsung Medical Center, Sungkyunkwan University School of Medicine, Seoul, South Korea

${ }^{2}$ Division of Endocrinology and Metabolism, Department of Medicine, Samsung Medical Center, Sungkyunkwan University School of Medicine, Seoul, South Korea

${ }^{3}$ Department of Otorhinolaryngology - Head and Neck Surgery, Samsung Medical Center, Sungkyunkwan University School of Medicine, Seoul, South Korea

\section{*Corresponding Author: Doo-Sik Kong, MD, PhD}

Department of Neurosurgery, Samsung Medical Center

Sungkyunkwan University School of Medicine

81 Irwon-ro, Gangnam-gu, Seoul 06351, South Korea

Phone: 82-2-3410-3499

Mobile phone: 82-10-9933-0732

E-mail: neurokong@gmail.com

ORCID: https://orcid.org/0000-0002-7519-3594

Keywords Hyperprolactinemia; prolactinoma; non-functioning pituitary adenomas; transsphenoidal surgery; pituitary surgery 


\begin{abstract}
Preoperative diagnosis of prolactinomas is critical because dopamine agonists have been regarded as a primary treatment. However, serum prolactin level alone is suboptimal for differentiating prolactinomas from hyperprolactinemia-causing non-functioning pituitary adenomas (NFPAs). By using the tumor size, we investigated a more effective parameter for the discrimination.

223 patients with pituitary lesions of the histologic diagnosis were retrospectively reviewed. The prolactinoma group $(\mathrm{n}=48)$ showed higher serum prolactin $(258.6 \mu \mathrm{g} / \mathrm{L})$ and smaller maximal diameter $(16.6 \mathrm{~mm})$ than those in the NFPA group $(\mathrm{n}=175)(44.4 \mu \mathrm{g} / \mathrm{L}$ and $23.9 \mathrm{~mm}$; both $\mathrm{p}$-values $<0.001)$.

Using receiver operating curve analyses, we compared diagnostic performances of serum prolactin levels (PRL), a ratio of PRL to maximal tumor diameter (PRL/MD; PDR1), and MD squared (PRL/(MD)²; PDR2) in preoperative diagnosis of prolactinomas. PDR2 exhibited better performance with the cutoff value of $0.83[\mu \mathrm{g} / \mathrm{L}] / \mathrm{mm}^{2}$ (area under the curve $[$ AUC $\left.]=0.945\right)$, compared to the PDR1 $(8.93[\mu \mathrm{g} / \mathrm{L}] / \mathrm{mm}$ with AUC 0.938) and PRL (99. $\mu \mathrm{g} / \mathrm{L}$ with AUC 0.910). In the external validation study, PDR2 still maintained superiority over PDR1 and PRL (Accuracy of 94.8\%, 91.8\%, and 75.8\%, respectively).

In conclusion, PDR2 may be an effective parameter for preoperative discrimination of prolactinomas from NFPAs with hyperprolactinemia, and contribute to select patients who benefit from medical treatment primarily.
\end{abstract}




\section{Introduction}

Prolactinoma is the most common type of pituitary adenoma (PA), accounting for $32-66 \%$ of all pituitary tumors requiring treatment. Current guidelines suggest the use of dopamine agonists (DAs) as a primary treatment for almost all spectra of prolactinomas, from microadenomas to giant prolactinomas (maximal diameter $[M D]>40 \mathrm{~mm})^{l}$. Surgical indicative of prolactinomas is usually the second option for the adenomas resistant to DAs at least 3 months after initiation or the patients who are intolerable to the complications of medication ${ }^{2,3}$. Thus the clinical diagnosis of prolactinomas before surgery is essential for treatment strategy.

However, the preoperative diagnosis of prolactinomas has been a matter of debate because of other pituitary pathologies accompanied by hyperprolactinemia. Hyperprolactinemia is defined as serum prolactin (PRL) levels above the upper reference limit (commonly $>20 \mu \mathrm{g} / \mathrm{L}$ in men and $>25 \mu \mathrm{g} / \mathrm{L}$ in women), with different physiological, pharmacological, and pathological causes ${ }^{4-6}$. Though prolactinoma is the most common cause of the prolactin hypersecretion in PAs, the "stalk section effect" of non-functional pituitary adenomas (NFPAs), the mechanical compression of the stalk blocks dopamine inhibition of lactotroph, makes it challenging to discriminate prolactinomas ${ }^{4,7-9}$.

Previous endocrinology guidelines suggested several ranges of hyperprolactinemia to distinguish between these two pathologies. Regarding the lactotroph tumor cells secreting PRL, the level of PRL secretion in prolactinomas was associated with tumor size ${ }^{10-12}$. Serum PRL levels $>500 \mu g / \mathrm{L}$ are generally always indicative of prolactinomas ${ }^{3,4}$. Macroadenomas (MD larger than $10 \mathrm{~mm}$ ) often exhibit serum PRL higher than $250 \mu \mathrm{g} / \mathrm{L}$ and even reach 20,000 $\mu \mathrm{g} / \mathrm{L}$ or more, while microprolactinomas (MD less than $10 \mathrm{~mm}$ ) commonly result in hyperprolactinemia with the range between 100-200 $\mu \mathrm{g} / \mathrm{L}^{4,10}$. Hyperprolactinemia of less than $100 \mu \mathrm{g} / \mathrm{L}$ is often related to the diagnostic uncertainty. Up to $25 \%$ of microprolactinomas present with hyperprolactinemia $<100 \mu g / \mathrm{L}^{4,13}$. Other studies have reported elevated PRL levels $<100 \mu g / \mathrm{L}$ with a solid pituitary macroadenoma are highly suspicious of an NFPA ${ }^{4,8}$. These variations make serum PRL alone to be insufficient for discriminate prolactinomas from NFPA.

The authors conducted a retrospective study to investigate novel parameters to improve the preoperative differentiation of these two pathologies. We firstly examined the different relationships of serum PRL and MD in prolactinomas and NFPAs. Using this property, the diagnostic performance of potential diagnostic parameters - the ratio of serum PRL to MD (PRL/MD; PDR1) ([ $\mu \mathrm{g} / \mathrm{L}] / \mathrm{mm})$ and MD squared (PRL/[MD] $]^{2}$; PDR2) $\left([\mu \mathrm{g} / \mathrm{L}] / \mathrm{mm}^{2}\right)$ - were compared to conventional serum PRL level alone $(\mu \mathrm{g} / \mathrm{L})$. 


\section{Results}

\section{Patient demographic data}

A total of 223 patients were included in the final analyses, with 175 patients in the NFPA group and 48 patients in the prolactinoma group. The descriptive characteristics of the two groups are compared in Table 1. There was no significant difference in the proportion of sex between the NFPA and prolactinoma groups ( $25.7 \%$ versus $31.2 \%$, respectively, $p$-value $>0.05$ ). The median age of the NFPA group was higher than that of the prolactinoma group (43.0 years versus 30.0 years, respectively, p-value $<0.01$ ).

Forty-seven (21.1\%) patients had no preoperative clinical symptoms or signs related to hyperprolactinemia or mass effect. Incidental diagnosis of adenoma was more common in the NFPA group than in the prolactinoma group (36 cases [20.6\%] and four cases [6.2\%] in each group, $p<0.05$ ). Hyperprolactinemia-related clinical symptoms were common in prolactinoma group (31 cases [64.6\%] vs 44 cases $[25.1 \%]$ in NFPA group, $\mathrm{p}<0.01)$. There was no significant difference in the incidence of hypopituitarism (12 cases [6.9\%] in the NPFA group versus no cases in the prolactinoma group) and pituitary apoplexy (10 cases [5.7\%] in the NFPA group vs 4 cases $(8.3 \%)$ in the prolactinoma group) between the two groups $(\mathrm{p}>0.05$, each). Preoperative medication of dopamine agonist was only administered with the prolactinoma group in 21 cases (43.8\%).

\section{Surgical indications of prolactinoma group}

Nineteen of the 48 (39.6\%) patients in the prolactinoma group underwent surgical resection of adenoma due to complications related to medical DA treatment. Among them, twelve (25.0\%) patients experienced medication resistance, including persistent hyperprolactinemia (three cases, 6.2\%) or its symptoms (four cases, $8.3 \%$ ), or no reduction of adenoma size (five cases, $10.4 \%$ ). The other seven cases $(14.5 \%)$ showed medical intolerance before surgery, despite a change in the dopamine agonist regimen.

Five $(10.4 \%)$ patients with prolactinomas were primarily treated with surgery due to preparation of pregnancy. This decision was based on the previous studies that DAs significantly increase the risk of pregnancy loss and of preterm birth ${ }^{14-16}$. Pituitary apoplexy with clinical symptoms were diagnosed in 4 patients $(8.3 \%)$, and these patients were treated with steroid medication and urgent decompression surgery. $20(41.6 \%)$ patients with prolactinomas were preoperatively diagnosed as NFPA by the physician's empirical determination from their size and serum prolactin levels. 


\section{Comparison of serum prolactin and the maximal adenoma diameter between NFPA and Prolactinoma groups}

Serum PRL and the maximal tumor diameter (MD) also showed significant differences between the two groups (Table 1). All cases in the NFPA group were macroadenomas (MD range: 10.8 - 57.1mm), while prolactinoma group comprised 12 cases (25\%) of microadenomas and 36 cases $(75 \%)$ of macroadenomas. The prolactinoma group showed higher median values of serum PRL $(258.6 \mu \mathrm{g} / \mathrm{L})$ and smaller tumor MD $(16.6 \mathrm{~mm}$ ) than those in the NFPA group (serum PRL, $44.4 \mu \mathrm{g} / \mathrm{L} ; \mathrm{p}<0.01$ and MD,23.9 mm; $>$ p 0.05). The median ratio of PDR1 and PDR2 was significantly higher in the prolactinoma group (PDR1; 21.18 [ $\mu \mathrm{g}$ $/ \mathrm{L}] / \mathrm{mm}$ and PDR2; $1.64[\mu \mathrm{g} / \mathrm{L}] / \mathrm{mm} 2)$ than NFPA group (PDR1; $1.84[\mu \mathrm{g} / \mathrm{L}] / \mathrm{mm}$ and PDR2; $0.07[\mu \mathrm{g}$ /L]/mm2, p-value $<0.001)$.

We further compared the serum PRL and MD parameters according to tumor size, particularly in the prolactinoma group (Table 2). Macroprolactinomas showed larger scales of both serum PRL levels and tumor MD than those of microprolactinomas, whereas the median value of both PDR1 and PDR2 parameters was no significant difference between the two groups ( $p$-value $=0.11$ ).

\section{Correlation between serum PRL and MD in prolactinoma or NFPA groups}

We conducted a correlation test between serum PRL levels and MD in each histological group. Pearson's correlation coefficients were used to estimate the linear relationship between the two variables. In the prolactinoma group, moderate strength of linear correlation between serum PRL and MD was confirmed in the positive direction (Pearson's $\mathrm{r}=0.43, \mathrm{p}=0.002)<0.05$ ). In comparison, a low correlation was confirmed (Pearson's $\mathrm{r}=0.17, \mathrm{p}=0.028$ ) in the NFPA group (Figure. 1).

\section{Diagnostic performance of PRL, PDR1, and PDR2 in the study group}

The receiver operating characteristic (ROC) curve and the diagnostic power of PRL, PDR1, and PDR1

were calculated (Figure 2). The optimal cutoff for indicating prolactinomas was determined using the Youden index, which maximizes the sum of sensitivity and specificity.

PDR2, a PRL to MD squared ratio (PRL/[MD]2), revealed the highest performance with the area under the curve (AUC) of $0.945(0.909-0.981)$ with the cutoff of $0.83[\mu \mathrm{g} / \mathrm{L}] / \mathrm{mm} 2$, providing a sensitivity of $81.2 \%$ 
and specificity of 97.1\%. PDR1, a ratio of PRL to MD (PRL/MD), demonstrated second-best results with the curve with an AUC of 0.938 (0.901-0.975) with a cutoff value of $8.93[\mu \mathrm{g} / \mathrm{L}] / \mathrm{mm}$. Meanwhile, PRL alone exhibited the AUC of $0.910(0.866-0.955)$ and cutoff of $99.43 \mu \mathrm{g} / \mathrm{L}$. This cutoff value was much different from a conventional serum prolactin level of $250 \mu \mathrm{g} / \mathrm{L}$ for predicting prolactinomas. The overall accuracy of PRL was weaker than those of PDR1 and PDR2 (PRL; 82.9\% versus PDR1; 89.7\% and PDR2 $93.3 \%)$.

The statistical difference between three AUCs were examined by the DeLong's test. The AUCs of PDR1 and PDR2 were significantly higher than PRL (PRL versus PDR1; $\mathrm{p}=0.012$ and PRL versus PDR2; $\mathrm{p}=0.037$ ). In contrast, there was no significant difference between the AUCs of PDR1 and PDR2 ( $\mathrm{p}=0.339)$. These results suggest that the ratio of serum prolactin to adenoma size showed better diagnostic powers than serum prolactin level alone in the study group.

\section{Optimal parameter for preoperative diagnosis of prolactinomas: validation with the test group}

We conducted external validation with an independent cohort of 50 patients to select the optimal parameters among three cutoffs from ROC analyses. The test group consisted of 13 patients with prolactinoma and 37 patients with NFPA. Table 1 described clinical characteristics and correlation between serum prolactin and tumor diameter.

In the validation group, PDR2 of $0.83[\mu \mathrm{g} / \mathrm{L}] / \mathrm{mm} 2$ retained best results performance (sensitivity of $92.3 \%$, specificity of $97.3 \%$, and the accuracy of $94.8 \%$ ) than PRL and PDR1 (overall accuracy of $75.9 \%$ and 91.8\%, respectively) (Figure 3).

Cohen's Kappa coefficient, representing the degree of agreement between reference classification (clinical diagnosis in our study) and new classifier (PRL, PDR1, and PDR2), also revealed strong consistency in PDR2 ( $k=0.896)$. PDR1 showed a moderate-to-strong degree kappa coefficient of 0.729 , while kappa of PRL alone was low $(\mathrm{k}=0.384)$. To further evaluate whether the statistical difference exists in classification between reference and new predictor, we used McNemar's test - PRL; $p=0.001$, PDR1; $p=0.041$ and PDR2; $\mathrm{p}=1.000$. As a $\mathrm{p}$-value more significant than 0.05 in McNemar's test suggests no significant difference between the two classifiers, the prediction of prolactinomas with PDR2 was statistically consistent with the reference. These results suggested that PDR2 is the optimal model among three parameters for differentiating prolactinomas from NFPAs. 


\section{Discussion}

This study investigated the predictive value of PRL and PDR for preoperative differentiation of prolactinomas and NFPAs. Distinguishing these two pathologies is critical given the satisfactory response to DAs in prolactinomas and the need for surgical resection in large NFPAs. The European Endocrine Society suggested serum PRL levels $>250 \mu \mathrm{g} / \mathrm{L}$ in macroadenomas (diameter $>1 \mathrm{~cm}$ ) as a clinical diagnostic threshold for prolactinomas ${ }^{3}$. In our data, this threshold was insufficient for the diagnosis of prolactinomas. $24(50 \%)$ of 48 patients with prolactinoma exhibited mild elevation of serum PRL levels $<$ $250 \mu \mathrm{g} / \mathrm{L}$ at the initial diagnosis, with 9 patients of microadenoma and 15 of macroadenoma. Five (10.4\%) of 48 patients presented with an even low degree of hyperprolactinemia with serum PRL levels $<100$ $\mu g / \mathrm{L}$.

NFPA and other sellar masses (growth hormone, adrenocorticotropic hormone, or thyroid-stimulating hormone; craniopharyngiomas; hypophysitis; etc.) are known to be typically associated with mild hyperprolactinemia $<100 \mu \mathrm{g} / \mathrm{L}^{3,13,17,18}$. These results were consistent with our results, as $142(81.1 \%)$ of 175 NFPAs in this study displayed a serum PRL level of less than $100 \mu \mathrm{g} / \mathrm{L}$. We found moderate-to-high levels of hyperprolactinemia in some patients with NFPA, as 27 (15.4\%) showed serum PRL levels between 100 and $250 \mu \mathrm{g} / \mathrm{L}$. Serum PRL levels of outliers, approximately $>1,000 \mu \mathrm{g} / \mathrm{L}$, were checked in three patients in the NPFA group, which all were macroadenoma with tumor diameter larger than $3 \mathrm{~cm}$. Thus the maximal PRL levels found in non-functional macroadenomas are still a matter of debate.

Previous studies reported that hormonal symptoms were much more prevalent than mass effects in prolactinomas and vice versa in NFPAs ${ }^{10,19}$. We found similar findings that hyperprolactinemic symptoms such as amenorrhea, galactorrhea was more common in prolactinomas $(62.6 \%$ in prolactinomas vs $25.1 \%$ in NFPAs, $\mathrm{p}<0.001$ ) and tumor mass effects with headache or visual disturbance were more prevalent in NFPAs $(50.9 \%$ in NFPAs vs. $29.2 \%$ in prolactinomas, $\mathrm{p}<0.05)$. These different clinical manifestations might help differentiate prolactinomas and NFPAs.

Tumor size and serum PRL levels displayed different relationships in our study. Prolactinomas showed a moderate linear correlation between serum PRL and tumor MD in the positive direction (Pearson's $r=0.43$, p-value $=0.002$ ), while NFPAs exhibited a weak correlation between the two parameters (Pearson's $r=0.17$,

$\mathrm{p}$-value $=0.028$ ). These results were comparable to other studies ${ }^{1,3,10,20}$, which suggest that lactotroph tissue is more contributable to the hyperprolactinemia than the stalk section effect due to the tumor mass size ${ }^{21-23}$.

Based on these results, several attempts were made to incorporate adenoma size into differentiating prolactinomas and hyperprolactinemia-causing NPFAs. Burke, et al. demonstrated the serum PRL cutoff values according to tumor volume of prolactinomas to distinguish them from NFPAs $(43.65 \mu \mathrm{g} / \mathrm{L}$ for $<0.5$ 
$\mathrm{cm}^{3}$ [MD < $1 \mathrm{~cm}$ ], $60.05 \mu \mathrm{g} / \mathrm{L}$ for 0.5 to $4 \mathrm{~cm}^{3}\left[\mathrm{MD}=1-2 \mathrm{~cm} \text { ], and } 248.15 \mu \mathrm{g} / \mathrm{L}>4 \mathrm{~cm}^{3}[\mathrm{MD}>2 \mathrm{~cm}]\right)^{11}$. Wright et al. used the ratio of serum PRL to tumor volume (PRL/V) for diagnosis of prolactinomas ( $\mathrm{n}=21)$ from NFPAs ( $\mathrm{n}=58)$, suggesting $21.62[\mathrm{ng} / \mathrm{mL}] / \mathrm{cm}^{3}$ as the cut off value with a sensitivity of $100 \%$ and specificity of $82.76 \%$. However, the statistical difference in diagnostic performance between the ROCs of PRL/V and serum PRL level alone was not described ${ }^{12}$. Further investigation is needed due to the smallsized sample and heterogeneous pathologies in the control group.

In this study, we examined and validated the diagnostic performance of the novel parameters, the ratio of PRL to MD (PDR1) and MD squared (PDR2), to that of PRL alone. The optimal cutoff values were $99.42 \mu \mathrm{g} / \mathrm{L}$ for PRL (AUC=0.910 and accuracy 82.9\%), $8.93[\mu \mathrm{g} / \mathrm{L}] / \mathrm{mm}$ for PDR1 (AUC=0.938, accuracy $=89.7 \%$ ) and $0.83[\mu \mathrm{g} / \mathrm{L}] / \mathrm{mm}^{2}$ for PDR2 (AUC=0.945 and accuracy 93.3\%). Both PDR models had superior outcomes in the ROC analyses than PRL, and in the validation study, the PDR2 model was the best classifier with statistical significance. These results suggested that considering the prolactinproductivity per adenoma size may improve the preoperative prediction of prolactinomas.

The limitation of this study includes the bias from its retrospective nature and sample sizes susceptible to outlier effects. One of the major limitations is selection bias; data were obtained only from surgical cases, causing relatively low "prevalence" of prolactinomas compared to NFPAs in our clinical setting. In the future, multi-center and prospective clinical studies are required to improve the accuracy and further elucidate the role of PDR in the differential diagnosis of prolactinomas from hyperprolactinemia-causing other pituitary pathologies.

In conclusion, our study demonstrated the effectiveness of serum PRL to tumor size ratio as a potential parameter for preoperative differentiation of prolactinomas and NFPAs. Based on the positive correlation between serum PRL and tumor MD in prolactinomas, contrary to the weak relationship observed in NFPAs, we examined and validated the diagnostic value of the PDR parameters compared to PRL alone. The optimal thresholds of the PRL to MD squared ratio may contribute to preoperative diagnosis of prolactinomas from other conditions of PAs, hence improving a treatment strategy whether administration of DA agonist or surgical resection should be recommended.

\section{Methods}

\section{Patient enrollment}

We performed a retrospective review of patients who underwent trans-sphenoidal surgery (TSS) for pituitary lesions between January 2015 and May 2021. This study was approved by the Samsung Medical 
Center institutional review board (IRB number: 2021-06-028). The informed consent was waived by Samsung Medical Center institutional review board, due to retrospective analyses. All medical performance in this study were conducted according to current diagnostic or treatment guidelines.

Among 997 consecutive patients with PAs who underwent TSS in this period, hyperprolactinemia, defined as a serum PRL level $>25 \mu \mathrm{g} / \mathrm{L}$, was confirmed at the time of initial diagnosis in 242 patients. We then identified the histopathology of these patients according to the World Health Organization (WHO) classification of pituitary tumors, using the version of 2004 and 2017 for patients who underwent TSS before 2017 and after 2017, respectively. Prolactinomas are defined as lactotroph adenomas that mainly express PRL and related hormonal symptoms or signs such as galactorrhea-amenorrhea syndrome. NFPAs are defined as PAs with a lack of clinical and biochemical evidence of adenohypophyseal hormone access. In the case of the diagnosis according to 2017 WHO classification, NFPAs included gonadotroph adenomas, silent corticotroph adenomas, silent adenomas of pit-1 derivation with no related hormonal excess and symptoms, and null cell adenomas. Finally, patients who were pathologically diagnosed as prolactinomas or NFPAs were included in the study. Other pathologies of PAs, including somatotroph adenoma, corticotroph adenoma, thyrotroph adenoma and plurihormonal adenoma were excluded.

Surgical indications of PAs were as follows: for prolactinomas, i) their adenomas were resistant to medical therapy at least 3-6 months after initiation, with persistent hormonal symptoms, elevated PRL levels, and constant or increased size of tumors, ii) patients are intolerable to the side effects of medical therapy, iii) patients who refused long term medications, due to preparation of pregnancy or preference for surgery, and iv) patients who were younger with PAs feasible for complete resection ${ }^{2,3,24}$. For NFPAs, patients who presented with non-PRL-related signs and symptoms or sign related to the mass effect of PAs were indicated to undergo surgery. The presence of tumor apoplexy, manifesting as severe headaches and a sharp decrease in vision, indicated surgical treatment in both types of adenomas ${ }^{25}$.

The preoperative serum PRL levels and maximal tumor diameters were obtained in all enrolled patients. Especially in prolactinomas, we selected the initial serum PRL levels before medical treatment to minimize the effect of medication on volume change. The maximal diameter of the tumor was measured in axial, coronal and sagittal planes of diagnostic sellar magnetic resonance images. The longest diameter of three measurements was defined as "MD (mm)" in this study. The correlation between serum prolactin and tumor size was verified. The experimental parameters, PRL/MD $([\mu \mathrm{g} / \mathrm{L}] / \mathrm{mm})(\mathrm{PDR} 1)$ and $\mathrm{PRL} /[\mathrm{MD}]^{2}\left([\mu \mathrm{g} / \mathrm{L}] / \mathrm{mm}^{2}\right)$ (PDR2) and serum PRL $(\mu \mathrm{g} / \mathrm{L})$ were calculated and their diagnostic performance were compared by ROC analyses. For external validation, an additional cohort was created independently from the study population. 
Among 64 patients who underwent TSS for hyperprolactinemia between June 2020 and May 2021, 50 cases of prolactinomas or NFPAs were identified and included in the validation group.

\section{Statistical analysis}

R 4.0.3 package (R Development Core Team, Oakland) was used for the statistical analyses. Comparisons between patients with prolactinoma and NFPA were performed in terms of general characteristics and relationships between tumor diameter and serum PRL levels. Pearson's correlation test was used to analyze the relationship between serum PRL level and tumor diameter according to each pathologic group. The diagnostic sensitivity, specificity, positive predictive value, negative predictive value, and the diagnostic accuracy of PRL, PDR1, and PDR2 were recorded. The accuracy was calculated as a conventional method used in model classification as below.

$(\mathrm{TP}+\mathrm{TN}) /(\mathrm{TP}+\mathrm{FP}+\mathrm{TN}+\mathrm{FN})$

$\mathrm{TP}=$ True positive, $\mathrm{TN}=$ True negative, $\mathrm{FP}=$ False positive, $\mathrm{FN}=$ False negative

ROC curves were used to investigate the optimal cutoff values of each parameter and AUC was compared using the student's t-test. We presented the $2 \mathrm{X} 2$ confusion matrix for validation results with Cohen's kappa coefficient and McNemar's test for the final selection of the best cutoff model. $\mathrm{P}<0.05$ was considered statistically significant. 


\section{Declarations}

Funding: This research did not receive any specific grant from funding agencies in the public, commercial, or not-for-profit sectors.

Conflicts of interest/Competing interests: The authors report no conflict of interest concerning the materials or methods used in this study or the findings specified in this paper.

\section{Author Contributions}

Jeong Hwa Kim: Conceptualization, Data curation, Formal Analysis, Writing - Original Draft, Writing Review and Editing;

Doo-Sik Kong : Conceptualization, Data Curation, Formal Analysis, Supervision, Writing - Original Draft, Writing - Review and Editing;

Kyu Yeon Hur, Sang Duk Hong: Conceptualization, Data curation, Writing - Review and Editing.

Jung Won Choi, Ho Jun Seol, Do-Hyun Nam and Jung-Il Lee: Data Curation, Supervision, Writing Review and Editing; 


\section{References}

1 Casanueva, F. F. et al. Guidelines of the Pituitary Society for the diagnosis and management of prolactinomas. Clin Endocrinol (Oxf) 65, 265-273, doi:10.1111/j.1365-2265.2006.02562.x (2006).

2 Iglesias, P. \& Díez, J. J. Macroprolactinoma: a diagnostic and therapeutic update. QJM: An International Journal of Medicine 106, 495-504, doi:10.1093/qjmed/hcs240 (2013).

3 Melmed, S. et al. Diagnosis and treatment of hyperprolactinemia: an Endocrine Society clinical practice guideline. J Clin Endocrinol Metab 96, 273-288, doi:10.1210/jc.2010-1692 (2011).

4 Vilar, L., Vilar, C. F., Lyra, R. \& Freitas, M. D. C. Pitfalls in the Diagnostic Evaluation of Hyperprolactinemia. Neuroendocrinology 109, 7-19, doi:10.1159/000499694 (2019).

5 Vilar, L. et al. Controversial issues in the management of hyperprolactinemia and prolactinomas An overview by the Neuroendocrinology Department of the Brazilian Society of Endocrinology and Metabolism. Arch Endocrinol Metab 62, 236-263, doi:10.20945/2359-3997000000032 (2018).

6 Wang, A. T. et al. Treatment of hyperprolactinemia: a systematic review and meta-analysis. Syst Rev 1, 33, doi:10.1186/2046-4053-1-33 (2012).

7 Skinner, D. C. Rethinking the stalk effect: a new hypothesis explaining suprasellar tumor-induced hyperprolactinemia. Med Hypotheses 72, 309-310, doi:10.1016/j.mehy.2008.08.030 (2009).

8 Zhang, F., Huang, Y., Ding, C., Huang, G. \& Wang, S. The prevalence of hyperprolactinemia in non-functioning pituitary macroadenomas. Int J Clin Exp Med 8, 18990-18997 (2015).

9 Ntali, G. \& Wass, J. A. Epidemiology, clinical presentation and diagnosis of non-functioning pituitary adenomas. Pituitary 21, 111-118, doi:10.1007/s11102-018-0869-3 (2018).

10 Chanson, P. \& Maiter, D. The epidemiology, diagnosis and treatment of Prolactinomas: The old and the new. Best Pract Res Clin Endocrinol Metab 33, 101290, doi:10.1016/j.beem.2019.101290 (2019).

11 Burke, W. T. et al. Prolactinomas and nonfunctioning adenomas: preoperative diagnosis of tumor type using serum prolactin and tumor size. Journal of neurosurgery 133, 321-328 (2019).

12 Wright, K. et al. Tumor volume improves preoperative differentiation of prolactinomas and nonfunctioning pituitary adenomas. Endocrine, 1-8 (2021).

13 Vilar, L. et al. Diagnosis and management of hyperprolactinemia: results of a Brazilian multicenter study with 1234 patients. $J$ Endocrinol Invest 31, 436-444, doi:10.1007/bf03346388 (2008).

14 Hurault-Delarue, C., Montastruc, J.-L., Beau, A.-B., Lacroix, I. \& Damase-Michel, C. Pregnancy outcome in women exposed to dopamine agonists during pregnancy: a pharmacoepidemiology study in EFEMERIS database. Archives of Gynecology and Obstetrics 290, 263-270, doi:10.1007/s00404-014-3210-z (2014). 
15 Glezer, A. \& Bronstein, M. D. Prolactinomas in pregnancy: considerations before conception and during pregnancy. Pituitary 23, 65-69, doi:10.1007/s11102-019-01010-5 (2020).

16 Tanrikulu, S. \& Yarman, S. Outcomes of Patients with Macroprolactinoma Desiring Pregnancy: Follow-Up to 23 Years from a Single Center. Horm Metab Res, doi:10.1055/a-1468-4608 (2021).

17 Molitch, M. E. Disorders of prolactin secretion. Endocrinology and metabolism clinics of North America 30, 585-610 (2001).

18 Molitch, M. E. Drugs and prolactin. Pituitary 11, 209-218 (2008).

19 Hong, J. W., Lee, M. K., Kim, S. H. \& Lee, E. J. Discrimination of prolactinoma from hyperprolactinemic non-functioning adenoma. Endocrine 37, 140-147 (2010).

20 Cander, S., Gül, Ö., Ertürk, E., Tuncel, E. \& Ersoy, C. Prolactin levels and gender are associated with tumour behaviour in prolactinomas but Ki-67 index is not. Endokrynol Pol 65, 210-216, doi:10.5603/ep.2014.0029 (2014).

21 Smith, M. V. \& Laws, E. R., Jr. Magnetic resonance imaging measurements of pituitary stalk compression and deviation in patients with nonprolactin-secreting intrasellar and parasellar tumors: lack of correlation with serum prolactin levels. Neurosurgery 34, 834-839; discussion 839, doi:10.1227/00006123-199405000-00007 (1994).

22 Zaidi, H. A. et al. Time Course of Resolution of Hyperprolactinemia After Transsphenoidal Surgery Among Patients Presenting with Pituitary Stalk Compression. World Neurosurg 97, 2-7, doi:10.1016/j.wneu.2016.09.066 (2017).

23 Lyu, L. et al. Hyperprolactinemia in clinical non-functional pituitary macroadenomas: A STROBEcompliant study. Medicine (Baltimore) 99, e22673, doi:10.1097/md.0000000000022673 (2020).

24 Chanson, P., Borson-Chazot, F., Chabre, O. \& Estour, B. Drug treatment of hyperprolactinemia. Annales d'Endocrinologie 68, 113-117, doi:https://doi.org/10.1016/j.ando.2007.03.003 (2007).

25 Chanson, P. et al. Management of clinically non-functioning pituitary adenoma. Ann Endocrinol (Paris) 76, 239-247, doi:10.1016/j.ando.2015.04.002 (2015). 


\section{Figure Captions}

Figure 1. Correlation between serum prolactin level and tumor diameter according to histologic groups.

Figure 2. Comparison of the ROC curves and diagnostic performance of serum prolactin level (PRL) and a ratio of serum prolactin to adenoma maximal diameter (PRL/MD, PDR1) and to diameter squared (PRL/[MD $]^{2}$, PDR2) for differentiating prolactinomas from non-functioning pituitary adenomas.

Figure 3. Validation of PRL, PDR1, and PDR2 classification models using confusion matrix.

A classification of prolactinomas (prl) and non-functional pituitary adenoma (nfpa) by clinicopatholgical diagnosis is the reference. Predictors are defined as classification by PRL, PDR1, and PDR2. The p-values of McNemar's test were described beside each matrix. 


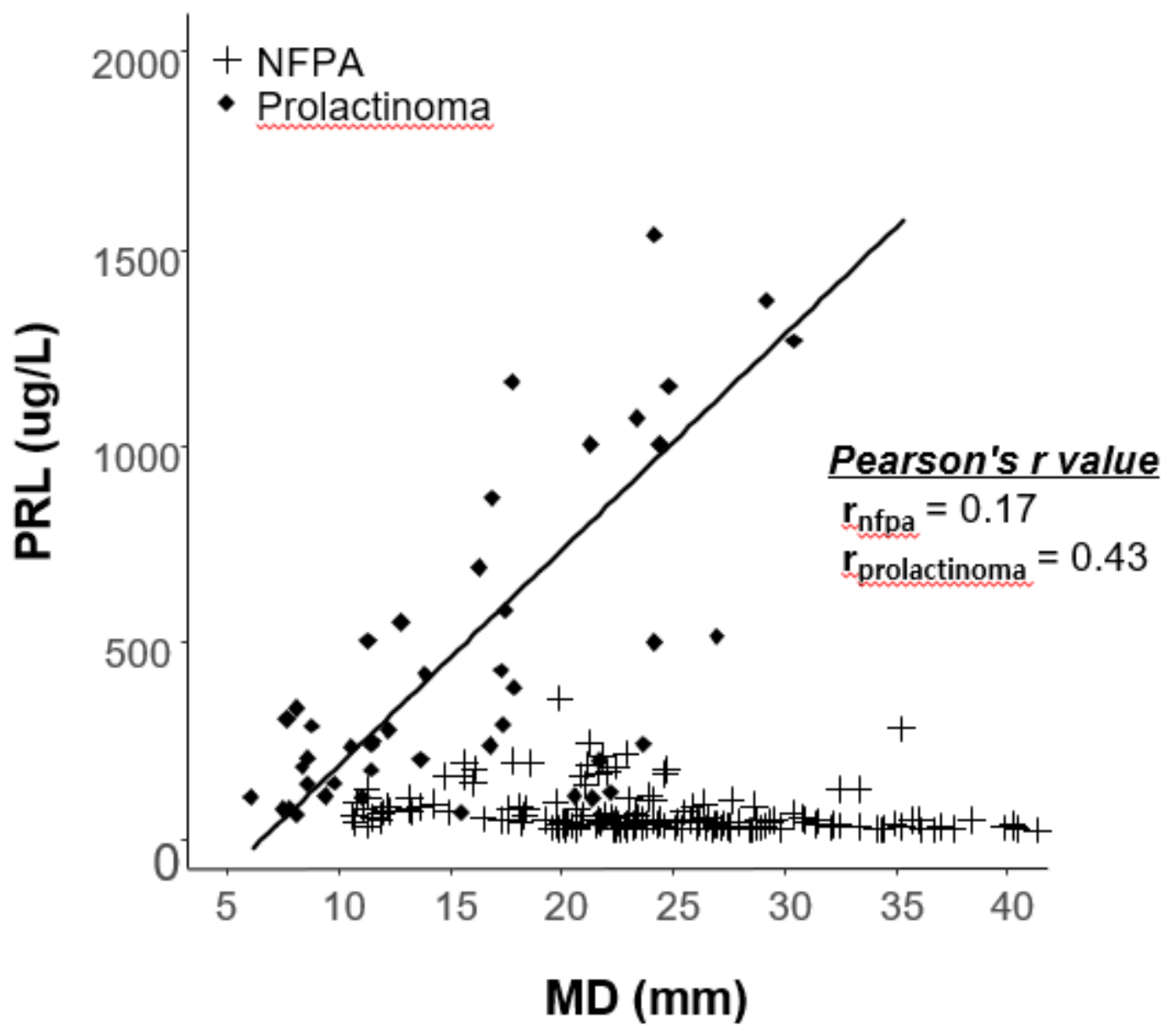

Figure 1

Correlation between serum prolactin level and tumor diameter according to histologic groups.

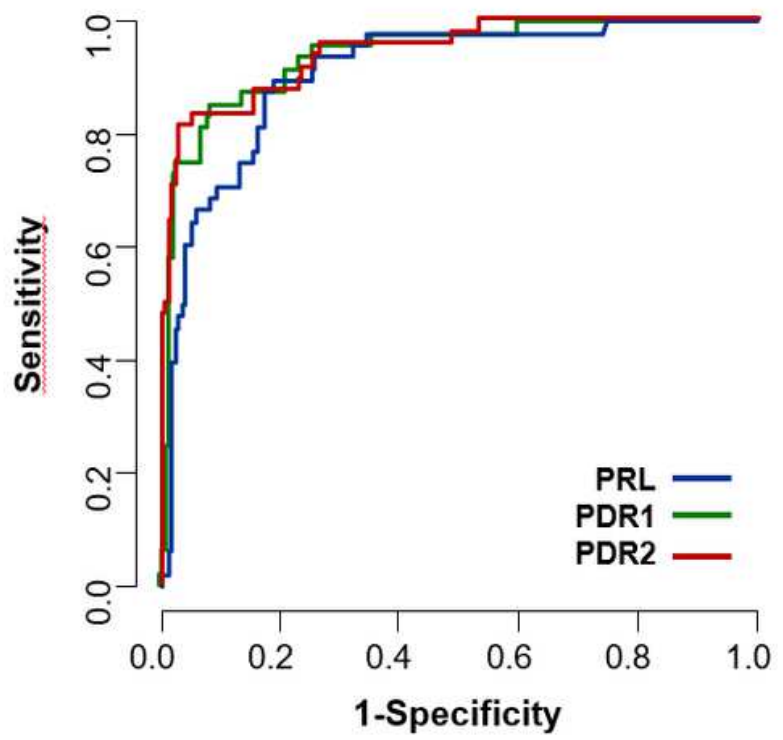

\begin{tabular}{|lccc|}
\hline \multicolumn{4}{c}{ Study group (n=223) } \\
\hline & $\begin{array}{c}\text { PRL } \\
\text { (ug/L) }\end{array}$ & $\begin{array}{c}\text { PDR1 } \\
([\mathrm{ug} / \mathrm{L}] / \mathrm{mm})\end{array}$ & $\begin{array}{c}\text { PDR2 } \\
\left([\mathrm{ug} / \mathrm{L}] / \mathrm{mm}^{2}\right)\end{array}$ \\
\hline Optimal cut off & 99.43 & 8.93 & 0.83 \\
\hline AUC & 0.910 & 0.938 & 0.945 \\
$(0.866-0.955)$ & $(0.901-0.975)$ & $(0.909-0.981)$ \\
\hline P-value & $<0.001^{\star}$ & $<0.001^{\star}$ & $<0.001^{\star}$ \\
\hline Sensitivity & $89.6 \%$ & $85.4 \%$ & $81.2 \%$ \\
\hline Specificity & $81.1 \%$ & $91.4 \%$ & $97.1 \%$ \\
\hline Positive predictive value & $56.6 \%$ & $71.9 \%$ & $88.6 \%$ \\
\hline Negative predictive value & $96.7 \%$ & $95.8 \%$ & $95.0 \%$ \\
\hline Accuracy & $82.9 \%$ & $89.7 \%$ & $93.3 \%$ \\
\hline
\end{tabular}


Figure 2

Comparison of the ROC curves and diagnostic performance of serum prolactin level (PRL) and a ratio of serum prolactin to adenoma maximal diameter (PRL/MD, PDR1) and to diameter squared (PRL/[MD]2, PDR2) for differentiating prolactinomas from non-functioning pituitary adenomas.

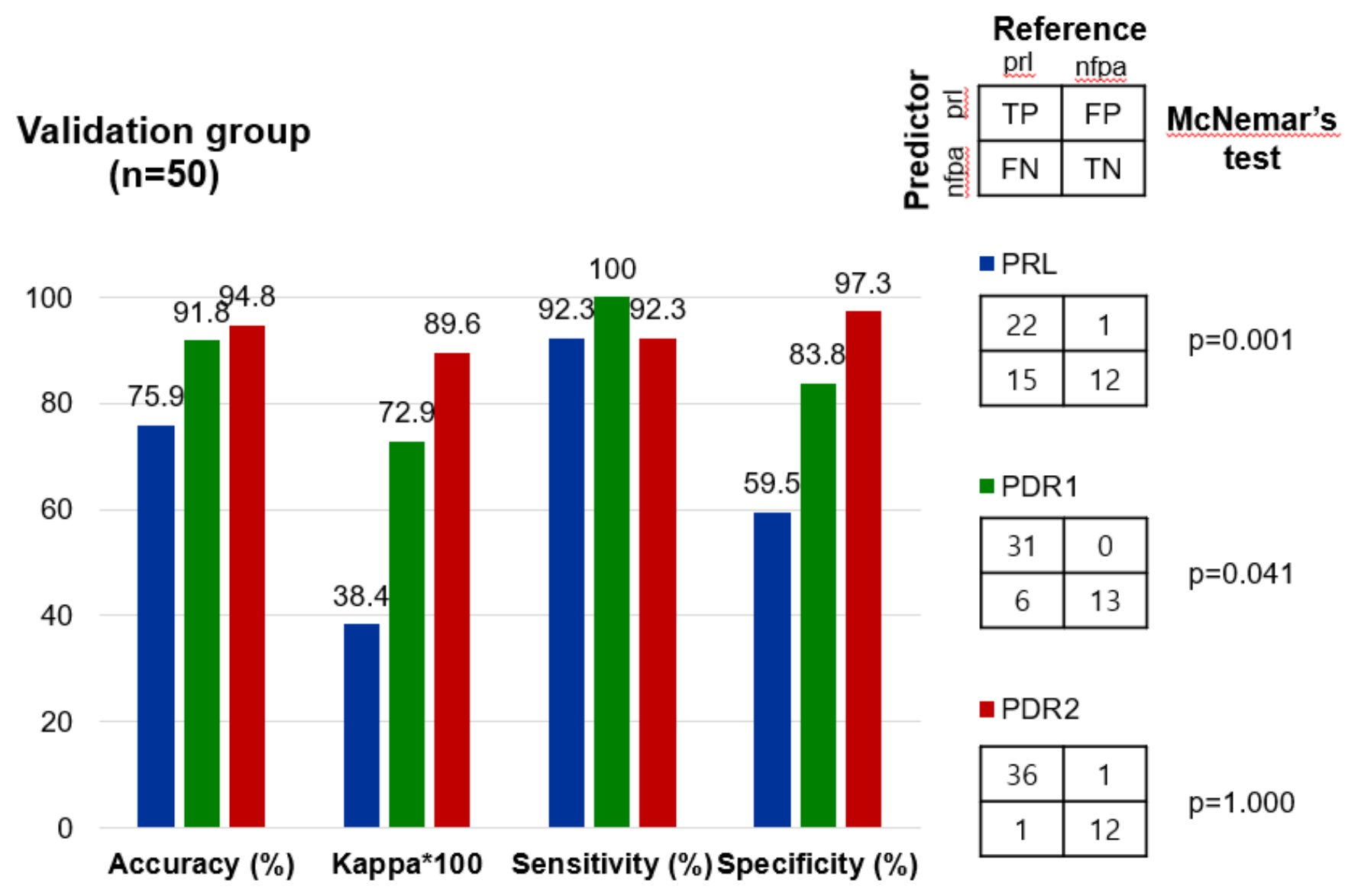

Figure 3

Validation of PRL, PDR1, and PDR2 classification models using confusion matrix. A classification of prolactinomas ( $\mathrm{prl}$ ) and non-functional pituitary adenoma ( $\mathrm{nfpa}$ ) by clinicopatholgical diagnosis is the reference. Predictors are defined as classification by PRL, PDR1, and PDR2. The p-values of McNemar's test were described beside each matrix. 